\title{
A Linear Matrix Inequality Approach to Robust Fault Detection Filter Design of Linear Systems with Mixed Time-Varying Delays and Nonlinear Perturbations
}

\author{
H.R. Karimi ${ }^{1}$, M. Zapateiro ${ }^{2}$, N. Luo $^{2}$ \\ ${ }^{1}$ Institute of Mechatronics, Department of Engineering, Faculty of Technology \\ and Science, University of Agder, N-4898 Grimstad, Norway \\ ${ }^{2}$ Institute of Informatics and Applications, University of Girona, \\ Campus de Montilivi, Ed. P4. 17071, Girona, Spain
}

\begin{abstract}
In this paper, the problem of robust fault detection filter (RFDF) design for a class of linear systems with some nonlinear perturbations and mixed neutral and discrete time-varying delays is investigated. By using a descriptor technique, Lyapunov-Krasovskii functional and a suitable change of variables, new required sufficient conditions are established in terms of delay-dependent linear matrix inequalities (LMIs) to synthesize the residual generation scheme. Based on Luenberger type observers, the explicit expression of the filters is derived for the fault such that both asymptotic stability and a prescribed level of disturbance attenuation are satisfied for all admissible nonlinear perturbations. A numerical example is provided to demonstrate the effectiveness and the applicability of the proposed method.
\end{abstract}

${ }^{1}$ Corresponding author. E-mail address: hamid.r.karimi@uia.no 


\section{INTRODUCTION}

There is an increasing demand for dynamic systems to become safer, more reliable and more economical in operation. This requirement extends beyond the normally accepted safety-critical systems e.g., nuclear reactors, aircraft and many chemical processes, to systems such as autonomous vehicles and some process control systems where the system availability is vital [37]. The field of fault diagnosis for dynamic systems (including fault detection and isolation) has become an important topic of research in the past three decades (see for instance [1]-[2], [5], [9], [11], [22] and the references therein). In the fault diagnosis scheme based on an adaptive observer, a fault cannot only be detected but also be approximated, and the fault estimate can be further used in fault-tolerant control. On the basis of an adaptive observer, Jiang et al. in [24] and Wang and Daley in [43] presented schemes that could deal with state fault only. Using the augmented error technique, Wang et al. in [44] proposed another scheme that could estimate actuator and sensor gain faults occurring simultaneously or sequentially. Combining adaptive observer with neural networks used as nonlinear function approximators, for instance, Demetriou and Polycarpou in [8] and Vemuri and Polycarpou in [41] developed an on-line learning scheme to detect and estimate faults for nonlinear systems. In [40], Trunov and Polycarpou generalized this scheme to detect and estimate both state and output faults in the presence of modelling uncertainties. Compared with the schemes of available in [24], [43]-[44], the one of the reference [40] is more general. On the other hand, time-delay exists widely in practice (see for instance [7], [21], [32], [35] and the references therein). Delay (or memory) systems represent a class of infinite-dimensional systems largely used to describe propagation and transport phenomena or population dynamics (see for instance [20], [29]-[30] and [33]). Delay differential systems are assuming an increasingly important role in many disciplines like economic, mathematics, science, and engineering. For instance, in economic systems, delays appear in a natural way since decisions and effects are separated by some time interval. The presence of a delay in a system may be the result of some essential simplification of the corresponding process model. The delay effects problem on the stability of systems including delays in the state and/or input is a problem of recurring interest since the delay presence may induce complex behaviours (oscillation, instability, bad performances) for the schemes (see for instance [21], [29], [33], [36] and [45]). Large delays in some reaction processes of chemical industries or time-delays induced by longdistance transportation and communication might cause the closed-loop systems unstable and deteriorate the control performance. On the contrary to the intensive investigation of robust fault diagnosis for uncertain systems and fault diagnosis for nonlinear systems, which have achieved much progress in recent years ([12] and [42]), the works on fault diagnosis for time-delay systems are very few. 
On the research of fault diagnosis for linear time-delay systems, Yang and Saif in [47] first proposed a scheme of actuator and sensor fault diagnosis using an unknown input observer and a technique of input estimation for systems with time-delays only in the state. In this work, modeling uncertainties were not considered and some assumptions on the system's structure decomposition were unreasonable. For systems with state and input time-delays, Ding et al. in [10] designed a robust fault detection filter that guaranteed both sensitivity to faults and insensitivity to disturbances. In the scheme of the reference [50], the influence of disturbances on the residual was further decreased using the idea of integrated design of $H_{\infty}$ filter and unknown input observer. Based on an adaptive observer, Jiang et al. in [24] developed a scheme to estimate abrupt state fault for linear (nonlinear) systems with only state time-delays, and no uncertainties were considered. For systems with constant time-delays in inputs and outputs only, Zhang et al., in [49] presented a state fault detection method based on parity space. Recently, a geometric approach for fault detection and isolation of retarded and neutral time-delay systems was developed in [34]. The time-delays investigated above are either in the state, the derivative of the state or in the input/output, neither in both of them or in the derivative of state. In practice, a system may involve time-delays in states, inputs/outputs and the derivative of states, and the influences of modeling uncertainties, noises and disturbances are perhaps not negligible (see for instance [3]-[4], [13], [17]-[19], [23], [26]-[28], [36], [39] and [46]). Furthermore, from the published results in [1]-[2], [9], [25] and [34], it appears that general results pertaining to robust fault detection of linear systems with mixed neutral and discrete time-varying delays, some nonlinear perturbations and an $H_{\infty}$ performance criteria, which are infinite dimensional systems in essence, are few and restricted, despite its practical importance, mainly due to the mathematical difficulties in dealing with such mixed delays and nonlinearities. Hence, it is our intention in this paper to tackle such an important yet challenging problem.

In this paper, we are concerned to develop a new delay-dependent stability criterion for robust fault detection filter (RFDF) problem of linear systems subjected to mixed neutral and discrete time-varying delays and some nonlinear perturbations which satisfy the Lipschitz conditions. The contribution of this paper is three-fold: first, this paper extends previous works on RFDF problem and derives some new theoretical results; second, this paper shows how the RFDF problem can be reduced to a convex problem with additional degrees of freedom to design a RFDF; third, by introducing a descriptor technique, using Lyapunov-Krasovskii functional and a suitable change of variables, we establish new required sufficient conditions in terms of delay-dependent linear matrix inequalities (LMIs) under which the desired RFDFs exist, and derive the explicit expression of these filters based on Luenberger type observers to satisfy both asymptotic stability and an $H_{\infty}$ performance condition. Furthermore, the incorporation of generated 
residual signal constraints increases the computational effort of the filter but also improves its estimation accuracy and the desired RFDF can be constructed through a convex optimization problem, which can be solved by using standard numerical algorithms. Finally, a numerical example is given to illustrate the proposed design method.

Notations. The superscript ' $T$ ' stands for matrix transposition; $\mathfrak{R}^{n}$ denotes the $n$-dimensional Euclidean space; $\mathfrak{R}^{n \times m}$ is the set of all real $m$ by $n$ matrices. $\|$.$\| refers to the Euclidean vector norm or the induced$ matrix 2-norm. $\operatorname{col}\{\cdots\}$ and $\operatorname{sym}(A)$ represent, respectively, a column vector and the matrix $A+A^{T}$. $\lambda_{\min }(A)$ and $\lambda_{\max }(A)$ denote, respectively, the smallest and largest eigenvalue of the square matrix $A$. The notation $P>0$ means that $P$ is real symmetric and positive definite; the symbol $*$ denotes the elements below the main diagonal of a symmetric block matrix. In addition, $L_{2}[0, \infty)$ is the space of squareintegrable vector functions over $[0, \infty)$. Matrices, if the dimensions are not explicitly stated, are assumed to have compatible dimensions for algebraic operations.

\section{PROBLEM DESCRIPTION}

We consider a class of continuous linear systems with some nonlinear perturbations and mixed neutral and discrete time-varying delays described by

$$
\left\{\begin{aligned}
\dot{x}(t)= & A x(t)+A_{1} x(t-\tau(t))+A_{2} \dot{x}(t-d(t))+B u(t)+E_{h_{1}} h_{1}(t, x(t))+E_{h_{2}} h_{2}(t, x(t-\tau(t))) \\
& \quad+E_{h_{3}} h_{3}(t, \dot{x}(t-d(t)))+E_{f} f(t)+B_{w} w(t) \\
x(t)= & \varphi(t) \quad t \in[-\kappa, 0] \\
z(t)= & C_{1} x(t) \\
y(t)= & C_{2} x(t)+D u(t)+C_{h} h_{4}(t, x(t))+C_{f} f(t)+D_{w} w(t)
\end{aligned}\right.
$$

where $\quad x(t) \in \mathfrak{R}^{n}, \quad w(t) \in L_{2}^{s}[0, \infty), \quad u(t) \in \mathfrak{R}^{m}, \quad z(t) \in \mathfrak{R}^{z}$ and $y(t) \in \mathfrak{R}^{p}$ are corresponded to state vector, disturbance input, control inputs, controlled outputs and measurements, respectively. The term $f(t) \in \mathfrak{R}^{l}$ corresponds to fault modes and $E_{f}$ is called fault signature which is assumed known. $h_{1}(t, x(t))$, $h_{2}(t, x(t-\tau(t))), h_{3}(t, \dot{x}(t-d(t)))$ and $h_{4}(t, x(t))$ are time-varying vector-valued functions which are unknown and present the nonlinear parameter perturbations. The time-varying function $\varphi(t)$ is continuous vector valued initial function and the parameters $\tau(t)$ and $d(t)$ are time-varying delays satisfying

$$
\begin{array}{cc}
0 \leq \tau(t) \leq \tau_{1}, & \dot{\tau}(t) \leq \tau_{2} \\
0 \leq d(t) \leq d_{1}, \quad \dot{d}(t) \leq d_{2}<1 .
\end{array}
$$

with $\kappa=\max \left\{\tau_{1}, d_{1}\right\}$.

One can define a difference operator $D: C\left([-\kappa, 0], \Re^{n}\right) \rightarrow \Re^{n}$ such that 


$$
D x_{t}=x(t)-A_{2} x(t-d(t))
$$

Definition 1. [21] The difference operator $D$ is said to be stable if the zero solution of the homogeneous difference equation $D x_{t}=0, t \geq 0, x_{0}=\Psi \in\{\Phi \in C([-\kappa, 0]): \nabla \Phi=0\}$ is uniformly asymptotically stable.

The stability of the difference operator $D$ is necessary for the stability of the system (1). Therefore, throughout the paper, the following assumption is needed to enable the application of Lyapunov's method for the stability of neutral systems.

A1) It follows from [21] that a delay-independent sufficient condition for the asymptotic stability of the system (1) is that all the eigenvalues of the matrix $A_{2}$ are inside the unit circle, i.e. $\lambda_{\max }\left(A_{2}\right)<1$.

Furthermore, we make the following assumption for the nonlinear perturbation functions in (1).

A2) The nonlinear function $h_{i}: \mathfrak{R} \times \mathfrak{R}^{n} \rightarrow \mathfrak{R}^{n}$ are continuous and satisfy $h_{i}(t, 0)=0$ and the Lipschitz condition, i.e., $\left\|h_{i}\left(t, x_{0}\right)-h_{i}\left(t, y_{0}\right)\right\| \leq\left\|U_{i}\left(x_{0}-y_{0}\right)\right\|$ for all $x_{0}, y_{0} \in \mathfrak{R}^{n}$ and $U_{i}$ are known matrices.

Remark 1. The model (1) can describe a large amount of well-known dynamical systems with timedelays, such as the delayed Logistic model, the chaotic models with time-delays, the artificial neural network models with time-delays, and the predator-prey model with delays (see for instance [16] and [48]).

In this paper, the authors' attention will be focused on the fault detection by a Luenberger type observer, the so-called robust fault detection filter (RFDF) with the following state-space equations

$$
\left\{\begin{aligned}
\dot{\hat{x}}(t)= & A \hat{x}(t)+A_{1} \hat{x}(t-\tau(t))+A_{2} \dot{\hat{x}}(t-d(t))+B u(t)+E_{h_{1}} h_{1}(t, \hat{x}(t)) \\
& +E_{h_{2}} h_{2}(t, \hat{x}(t-\tau(t)))+E_{h_{3}} h_{3}(t, \hat{x}(t-d(t)))+H(y(t)-\hat{y}(t)) \\
\hat{x}(t)= & \quad t \in[-\kappa, 0] \\
\hat{z}(t)= & C_{1} \hat{x}(t) \\
\hat{y}(t)= & C_{2} \hat{x}(t)+D u(t)+C_{h} h_{4}(t, \hat{x}(t)) \\
r(t)= & V(y(t)-\hat{y}(t))
\end{aligned}\right.
$$

where $r(t)$ is the so-called generated residual signal and the state-space matrices $H, V$ of the appropriate dimensions are the filter design objectives to be determined. In the absence of $w(t)$ and $f(t)$, it is required that

$$
\|x(t)-\hat{x}(t)\|_{2} \rightarrow 0 \quad \text { as } \quad t \rightarrow \infty
$$

where $\hat{x}(t) \in \mathfrak{R}^{n}$ is the estimation of $x(t)$ and $e(t)=\left[e_{1}(t), e_{2}(t), \ldots, e_{n}(t)\right]^{T}=x(t)-\hat{x}(t)$ is the estimation error. Then, the error dynamics between (1) and (4) can be expressed by 


$$
\left\{\begin{array}{l}
\dot{e}(t)=\left(A-H C_{2}\right) e(t)+A_{1} e(t-\tau(t))+A_{2} \dot{e}(t-d(t))+E_{h_{1}} \psi_{1}(t, e(t)) \\
\quad+E_{h_{2}} \psi_{2}(t, e(t-\tau(t)))+E_{h_{3}} \psi_{3}(t, \dot{e}(t-d(t)))-H C_{h} \psi_{4}(t, e(t))+\hat{B} \hat{w}(t) \\
z(t)-\hat{z}(t)=C_{1} e(t) \\
r(t)=V\left(C_{2} e(t)+C_{h} \psi_{4}(t, e(t))+\hat{D} \hat{w}(t)\right)
\end{array}\right.
$$

where $\quad \hat{w}(t):=\operatorname{col}\{w(t), f(t)\}, \quad \hat{D}:=\left[D_{w}, C_{f}\right], \quad \hat{B}:=\left[B_{w}-H D_{w}, E_{f}-H C_{f}\right], \quad \psi_{1}(t, e(t))=h_{1}(t, x(t))-$ $h_{1}(t, x(t)-e(t)), \psi_{2}(t, e(t-\tau(t)))=h_{2}(t, x(t-\tau(t)))-h_{2}(t, x(t-\tau(t))-e(t-\tau(t))), \psi_{3}(t, \dot{e}(t-d(t)))=h_{3}(t, \dot{x}(t-d(t)))-$ $h_{3}(t, \dot{x}(t-d(t))-\dot{e}(t-d(t)))$ and $\psi_{4}(t, e(t))=h_{4}(t, x(t))-h_{4}(t, x(t)-e(t))$.

From A2), the Mean Value theorem and the Leibniz-Newton formula, i.e., $e(t)-e(t-\tau(t))=\int_{t-\tau(t)}^{t} \dot{e}(s) d s$, it is easy to see

$$
\begin{aligned}
\psi_{2}(t, e(t))-\psi_{2}(t, e(t-\tau(t))) & =\dot{\psi}_{2}(\xi)(e(t)-e(t-\tau(t))) \\
& =\dot{\psi}_{2}(\xi) \int_{t-\tau(t)}^{t} \dot{e}(s) d s
\end{aligned}
$$

where $\xi$ is a point on the straight line between $e(t)$ and $e(t-\tau(t))$, which may be different for different rows of $\dot{\psi}_{2}(\xi)$.

Remark 2. It is noting that, from the equation (7), one can obtain

$$
\begin{aligned}
\left\|\psi_{2}(t, e(t))-\psi_{2}(t, e(t-\tau(t)))\right\| & =\left\|\dot{\psi}_{2}(\xi)(e(t)-e(t-\tau(t)))\right\| \\
& \leq\left\|\dot{\psi}_{2}(\xi)\right\|\|e(t)-e(t-\tau(t))\|
\end{aligned}
$$

thus, the Lipschitz constant of $\psi_{2}($.$) can be estimated by \max _{\xi}\left\|\dot{\psi}_{2}(\xi)\right\|$.

Therefore, from the equation (7), the estimation dynamics (6) can be represented in a descriptor model form as

$$
\left\{\begin{array}{l}
\dot{e}(t)=\eta(t) \\
\eta(t)=\left(A+A_{1}-H C_{2}\right) e(t)-\left(A_{1}+E_{h_{2}} S\right) \int_{t-\tau(t)}^{t} \eta(s) d s+A_{2} \eta(t-d(t))+E_{h_{1}} \psi_{1}(t, e(t)) \\
\quad+E_{h_{2}} \psi_{2}(t, e(t))+E_{h_{3}} \psi_{3}(t, \eta(t-d(t)))-H C_{h} \psi_{4}(t, e(t))+\hat{B} \hat{w}(t)
\end{array}\right.
$$

where $S:=\dot{\psi}_{2}(\xi)$.

Definition 2. The RFDF error dynamics (4) is said

1. to achieve asymptotic stability in the Lyapunov sense for $\hat{w}(t)=0$ if the error dynamics (6) are asymptotically stable for all admissible nonlinear perturbations.

2. to guarantee $H_{\infty}$ performance condition if under zero initial conditions, 


$$
\sup _{\|\hat{w}\|_{2} \neq 0} \frac{\left\|\left[\begin{array}{c}
z(t)-\hat{z}(t) \\
r(t)
\end{array}\right]\right\|_{2}}{\|\hat{w}(t)\|_{2}} \leq \gamma
$$

holds for all bounded energy disturbances and a prescribed positive value $\gamma$.

The fault detection problem we address here is as follows: Given a prescribed level of disturbance attenuation $\gamma>0$, find the RFDF of the type (4) in the sense of Definition 2.

Before ending this section, we recall a well-known lemma, which will be used in the proof of our main results.

Lemma 1. [36] For any arbitrary column vectors $a(t), b(t)$, matrices $\Phi(t), H, U$ and $W$ the following inequality holds:

$$
-2 \int_{t-r}^{t} a(s)^{T} \Phi(s) b(s) d s \leq \int_{t-r}^{t}\left[\begin{array}{l}
a(s) \\
b(s)
\end{array}\right]^{T}\left[\begin{array}{cc}
H & U-\Phi(s) \\
* & W
\end{array}\right]\left[\begin{array}{l}
a(s) \\
b(s)
\end{array}\right] d s
$$

where $\left[\begin{array}{cc}H & U \\ * & W\end{array}\right] \geq 0$.

\section{RFDF DESIGN}

In this section, the fault diagnosis problem presented in the previous section is investigated such that a sufficient condition is derived for the existence of the RFDF (4). The approach employed here is to develop a criterion for the existence of such filters is based on the LMI approach combined with the Lyapunov method. In the literature, extensions of the quadratic Lyapunov functions to the quadratic Lyapunov-Krasovskii functionals have been proposed for time-delayed systems (see for instance the Refs. [6], [14], [16], [20], [31], [35] and the references therein). We choose a Lyapunov-Krasovskii functional candidate for the error dynamics (6a) as

$$
V(t)=V_{1}(t)+V_{2}(t)+V_{3}(t)
$$

where

$$
\begin{gathered}
V_{1}(t)=e(t)^{T} P_{1} e(t)=\bar{\eta}(t)^{T} T P \bar{\eta}(t), \\
V_{2}(t)=\int_{t-\tau(t)}^{t} e(s)^{T} Q_{1} e(s) d s+\int_{t-d(t)}^{t} \eta(s)^{T} Q_{2} \eta(s) d s, \\
V_{3}(t)=\int_{t-\tau_{1}}^{t} \int_{s}^{t} \eta(\theta)^{T}\left(Q_{3}+Q_{4}\right) \eta(\theta) d \theta d s,
\end{gathered}
$$

with $\bar{\eta}(t):=\operatorname{col}\{e(t), \eta(t)\}, T:=\operatorname{diag}\{I, 0\}$ and 


$$
P:=\left[\begin{array}{cc}
P_{1} & 0 \\
P_{3} & P_{2}
\end{array}\right], P_{1}=P_{1}^{T}>0
$$

In the following theorem, we state our main results.

Theorem 1. Under A1)-A2), consider system (1)-(2) and let the scalars $\gamma, \tau_{1}, d_{1}>0, \tau_{2}, d_{2}, \varepsilon$ be given. If there exist the matrices $P_{2}, V, \hat{H}, U, M_{1}, \cdots, M_{8}$ and the positive definite matrices $P_{1}, H_{1}, Q_{1}, \cdots, Q_{4}$, satisfying the following LMIs

$$
\begin{aligned}
& {\left[\begin{array}{ccccccccc}
\Pi_{11} & \Pi_{12} & \Pi_{13} & \Pi_{14} & \Pi_{15} & \Pi_{16} & \Pi_{17} & \Pi_{18} & J^{T} C_{2}^{T} V^{T} \\
* & \Pi_{22} & -M_{3}^{T} & -M_{4}^{T} & -M_{5}^{T} & -M_{6}^{T} & -M_{7}^{T} & -M_{8}^{T} & 0 \\
* & * & \Pi_{33} & 0 & 0 & 0 & 0 & 0 & 0 \\
* & * & * & -I & 0 & 0 & 0 & 0 & 0 \\
* & * & * & * & -I & 0 & 0 & 0 & 0 \\
* & * & * & * & * & -I & 0 & 0 & 0 \\
* & * & * & * & * & * & -I & 0 & C_{h}^{T} V^{T} \\
* & * & * & * & * & * & * & -I & \hat{D}^{T} V^{T} \\
* & * & * & * & * & * & * & * & -I
\end{array}\right]<0,} \\
& {\left[\begin{array}{cc}
H_{1} & U \\
* & Q_{3}
\end{array}\right] \geq 0,}
\end{aligned}
$$

with $J:=[I, 0], \hat{J}:=[0, I], \Lambda_{1}:=Q_{1}+C_{1}^{T} C_{1}+U_{1}^{T} U_{1}+U_{4}^{T} U_{4}, \Lambda_{2}:=Q_{2}+\tau_{1} Q_{3}+d_{1} Q_{4}$ and

$$
\begin{gathered}
\Pi_{12}=-U+\left(\varepsilon J^{T}+\hat{J}^{T}\right) P_{2}^{T} A_{1}-M_{1}+J^{T} M_{2}^{T}, \Pi_{13}=\left(\varepsilon J^{T}+\hat{J}^{T}\right) P_{2}^{T} A_{2}, \\
\Pi_{14}=\left(\varepsilon J^{T}+\hat{J}^{T}\right) P_{2}^{T} E_{h_{1}}, \Pi_{15}=\left(\varepsilon J^{T}+\hat{J}^{T}\right) P_{2}^{T} E_{h 2}, \\
\Pi_{16}=\left(\varepsilon J^{T}+\hat{J}^{T}\right) P_{2}^{T} E_{h 3}, \Pi_{17}=-\left(\varepsilon J^{T}+\hat{J}^{T}\right) \hat{H} C_{h}, \\
\Pi_{18}=\left(\varepsilon J^{T}+\hat{J}^{T}\right) P_{2}^{T} \hat{B}, \Pi_{22}=-\left(1-\tau_{2}\right) Q_{1}+U_{2}^{T} U_{2}-\operatorname{sym}\left\{M_{2}\right\}, \Pi_{33}=-\left(1-d_{2}\right) Q_{2}+U_{3}^{T} U_{3}, \\
\Pi_{11}=\operatorname{sym}\left\{\left[\begin{array}{cc}
\varepsilon\left(P_{2}^{T} A-\hat{H} C_{2}\right) & P_{1}-\varepsilon P_{2}^{T} \\
P_{2}^{T} A-\hat{H} C_{2} & -P_{2}^{T}
\end{array}\right]\right\}+\operatorname{sym}\left\{U J-M_{1} J\right\}+\tau_{1} H_{1}+\operatorname{diag}\left\{\Lambda_{1}, \Lambda_{2}\right\} .
\end{gathered}
$$

Then there exists a RFDF of the type (4) which achieve the asymptotic stability and the $H_{\infty}$ performance condition, simultaneously, in the sense of Definition 2. Moreover, the matrix $H$ of the RFDF can be found by computing $H:=\left(P_{2}^{T}\right)^{-1} \hat{H}$.

Proof. Differentiating $V_{1}(t)$ in $t$ along the trajectory of the error dynamics (6a) we obtain 


$$
\begin{aligned}
\dot{V}_{1}(t)= & 2 e(t)^{T} P_{1} \dot{e}(t)=2 \bar{\eta}(t)^{T} \quad P^{T}\left[\begin{array}{c}
\dot{e}(t) \\
0
\end{array}\right] \\
= & 2 \bar{\eta}(t)^{T} P^{T}\left[\begin{array}{c}
\eta(t) \\
{[2,1]}
\end{array}\right] \\
= & 2 \bar{\eta}(t)^{T} P^{T}\left(\bar{A} \bar{\eta}(t)+\hat{J}^{T} A_{2} \eta(t-d(t))+\hat{J}^{T} E_{h_{1}} \psi_{1}(t, e(t))+\hat{J}^{T} E_{h_{2}} \psi_{2}(t, e(t))\right. \\
& \left.+\hat{J}^{T} E_{h_{3}} \psi_{3}(t, \eta(t-d(t)))-\hat{J}^{T} H C_{h} \psi_{4}(t, e(t))+\hat{J}^{T} \hat{B} \hat{w}(t)\right)+\beta_{1}(t)
\end{aligned}
$$

where $\bar{A}:=\left[\begin{array}{cc}0 & I \\ A+A_{1}-H C_{2} & -I\end{array}\right], \beta_{1}(t)=-2 \bar{\eta}(t)^{T} P^{T} \hat{J}^{T}\left(A_{1}+E_{h_{2}} S\right) \int_{t-\tau(t)}^{t} \eta(s) d s$ and

$$
\begin{aligned}
{[2,1]=} & -\eta(t)+\left(A+A_{1}-H C_{2}\right) e(t)-\left(A_{1}+E_{h_{2}} S\right) \int_{t-\tau(t)}^{t} \eta(s) d s+A_{2} \eta(t-d(t))+E_{h_{1}} \psi_{1}(t, e(t)) \\
& +E_{h_{2}} \psi_{2}(t, e(t))+E_{h_{3}} \psi_{3}(t, \eta(t-d(t)))-H C_{h} \psi_{4}(t, e(t))
\end{aligned}
$$

By Lemma 1 and (7), it is clear that

$$
\begin{aligned}
\beta_{1}(t) \leq & \int_{t-\tau(t)}^{t}\left[\begin{array}{c}
\bar{\eta}(t) \\
\eta(s)
\end{array}\right]^{T}\left[\begin{array}{cc}
H_{1} & U-P^{T} \hat{J}^{T}\left(A_{1}+E_{h_{2}} S\right) \\
* & Q_{3}
\end{array}\right]\left[\begin{array}{l}
\bar{\eta}(t) \\
\eta(s)
\end{array}\right] d s \\
\leq & \int_{t-\tau_{1}}^{t} \eta(s)^{T} Q_{3} \eta(s) d s+\tau_{1} \bar{\eta}(t)^{T} H_{1} \bar{\eta}(t)+2 \bar{\eta}(t)^{T}\left(U-P^{T} \hat{J}^{T} A_{1}\right)(e(t)-e(t-\tau(t))) \\
& \quad-2 \bar{\eta}(t)^{T} P^{T} \hat{J}^{T} E_{h_{2}}\left(\psi_{2}(t, e(t))-\psi_{2}(t, e(t-\tau(t)))\right)
\end{aligned}
$$

subject to the LMI (13b). The time derivative of the second and third terms of $V(t)$ are, respectively, as

$$
\begin{gathered}
\dot{V}_{2}(t)=e(t)^{T} Q_{1} e(t)-(1-\dot{\tau}(t)) e(t-\tau(t))^{T} Q_{1} e(t-\tau(t)) \\
\quad+\eta(t)^{T} Q_{2} \eta(t)-(1-\dot{d}(t)) \eta(t-d(t))^{T} Q_{2} \eta(t-d(t)) \\
\leq e(t)^{T} Q_{1} e(t)-\left(1-\tau_{2}\right) e(t-\tau(t))^{T} Q_{1} e(t-\tau(t)) \\
+\eta(t)^{T} Q_{2} \eta(t)-\left(1-d_{2}\right) \eta(t-d(t))^{T} Q_{2} \eta(t-d(t))
\end{gathered}
$$

and

$$
\dot{V}_{3}(t)=\tau_{1} \eta(t)^{T}\left(Q_{3}+Q_{4}\right) \eta(t)-\int_{t-\tau_{1}}^{t} \eta(s)^{T}\left(Q_{3}+Q_{4}\right) \eta(s) \quad d s
$$

Construct a HJI function in the form of

$$
J[e(t), \hat{w}(t)]=\frac{d}{d t} V(t)+\left[\begin{array}{c}
z(t)-\hat{z}(t) \\
r(t)
\end{array}\right]^{T}\left[\begin{array}{c}
z(t)-\hat{z}(t) \\
r(t)
\end{array}\right]-\gamma^{2} \hat{w}(t)^{T} \hat{w}(t)
$$

where derivative of $V(t)$ is evaluated along the trajectory of the error dynamics (6a). It is well known that the performance condition (10) is that the inequality $J[e(t), \hat{w}(t)]<0$ for every $\hat{w}(t) \in L_{2}^{s}[0, \infty)$ results in a function $V(t)$, which is strictly radially unbounded (see for instance [51]).

From (14)-(18) we obtain 


$$
\begin{aligned}
J[e(t), \hat{w}(t)]= & 2 \bar{\eta}(t)^{T} P^{T}\left(\bar{A} \bar{\eta}(t)+\hat{J}^{T} A_{2} \eta(t-d(t))+\hat{J}^{T} E_{h_{1}} \psi_{1}(t, e(t))+\hat{J}^{T} E_{h_{2}} \psi_{2}(t, e(t-\tau(t)))\right. \\
& \left.+\hat{J}^{T} E_{h_{3}} \psi_{3}(t, \eta(t-d(t)))-\hat{J}^{T} H C_{h} \psi_{4}(t, e(t))+\hat{J}^{T} \hat{B} \hat{w}(t)\right)+\tau_{1} \bar{\eta}(t)^{T} H_{1} \bar{\eta}(t) \\
& +2 \bar{\eta}(t)^{T}\left(U-P^{T} \hat{J}^{T} A_{1}\right)(e(t)-e(t-\tau(t)))+e(t)^{T}\left(C_{1}^{T} C_{1}+Q_{1}+C_{2}^{T} V^{T} V C_{2}\right) e(t) \\
& -\left(1-\tau_{2}\right) e(t-\tau(t))^{T} Q_{1} e(t-\tau(t))-\left(1-d_{2}\right) \eta(t-d(t))^{T} Q_{2} \eta(t-d(t)) \\
& +\eta(t)^{T} \Lambda_{2} \eta(t)-\int_{t-d_{1}}^{t} \eta(s)^{T} Q_{4} \eta(s) d s+\hat{w}(t)^{T}\left(\hat{D}^{T} V^{T} V \hat{D}-\gamma^{2} I\right) \hat{w}(t) \\
& +\psi_{4}(t, e(t))^{T} C_{h}^{T} V^{T} V C_{h} \psi_{4}(t, e(t))+\operatorname{sym}\left(e(t)^{T} C_{2}^{T} V^{T} V C_{h} \psi_{4}(t, e(t))\right) \\
& +\operatorname{sym}\left(e(t)^{T} C_{2}^{T} V^{T} V \hat{D} \hat{w}(t)\right)+\operatorname{sym}\left(\psi_{4}(t, e(t))^{T} C_{h}^{T} V^{T} V \hat{D} \hat{w}(t)\right)
\end{aligned}
$$

Using A2), we have

$$
\begin{gathered}
0 \leq-\psi_{1}(t, e(t))^{T} \psi_{1}(t, e(t))+e(t)^{T} U_{1}^{T} U_{1} e(t) \\
0 \leq-\psi_{2}(t, e(t-\tau(t)))^{T} \psi_{2}(t, e(t-\tau(t)))+e(t-\tau(t))^{T} U_{2}^{T} U_{2} e(t-\tau(t)) \\
0 \leq-\psi_{3}(t, \eta(t-d(t)))^{T} \psi_{3}(t, \eta(t-d(t)))+\eta(t-d(t))^{T} U_{3}^{T} U_{3} \eta(t-d(t)) \\
0 \leq-\psi_{4}(t, e(t))^{T} \psi_{4}(t, e(t))+e(t)^{T} U_{4}^{T} U_{4} e(t)
\end{gathered}
$$

Moreover, from the Leibniz-Newton formula and (6a), the following equation holds for any matrix $M$ with appropriate dimension,

$$
2 v(t)^{T} M\left(e(t)-e(t-\tau(t))-\int_{t-\tau(t)}^{t} \eta(s) d s\right)=0
$$

where $M:=\operatorname{col}\left\{M_{1}, M_{2}, \cdots, M_{8}\right\}$ and

$$
\vartheta(t):=\operatorname{col}\left\{\bar{\eta}(t), e(t-\tau(t)), \eta(t-d(t)), \psi_{1}(t, e(t)), \psi_{2}(t, e(t-\tau(t))), \psi_{3}(t, \eta(t-d(t))), \psi_{4}(t, e(t)), \hat{w}(t)\right\}
$$

By adding the right- and the left- hand sides of (20)-(21), respectively, to (19) and using the property $\int_{t-\tau_{1}}^{t-\tau(t)} \eta(s)^{T} Q_{4} \eta(s) \quad d s \geq 0$, it follows that

$$
J[e(t), \hat{w}(t)] \leq \vartheta(t)^{T}\left(\hat{\Pi}+\tau_{1} M Q_{4}^{-1} M^{T}\right) \vartheta(t)-\int_{t-\tau(t)}^{t}\left(\vartheta(t)^{T} M+\eta(s)^{T} Q_{4}\right) Q_{4}^{-1}\left(\vartheta(t)^{T} M+\eta(s)^{T} Q_{4}\right)^{T} d s
$$

where the matrix $\hat{\Pi}$ is given by

$$
\hat{\Pi}=\left[\begin{array}{cccccccc}
\hat{\Pi}_{11} & \hat{\Pi}_{12} & P^{T} \hat{J}^{T} A_{2} & P^{T} \hat{J}^{T} E_{h_{1}} & P^{T} \hat{J}^{T} E_{h_{2}} & P^{T} \hat{J}^{T} E_{h_{3}} & \hat{\Pi}_{17} & \hat{\Pi}_{18} \\
* & \hat{\Pi}_{22} & -M_{3}^{T} & -M_{4}^{T} & -M_{5}^{T} & -M_{6}^{T} & -M_{7}^{T} & -M_{8}^{T} \\
* & * & \hat{\Pi}_{33} & 0 & 0 & 0 & 0 & 0 \\
* & * & * & -I & 0 & 0 & 0 & 0 \\
* & * & * & * & -I & 0 & 0 & 0 \\
* & * & * & * & * & -I & 0 & 0 \\
* & * & * & * & * & * & \hat{\Pi}_{77} & \hat{\Pi}_{78} \\
* & * & * & * & * & * & * & \hat{\Pi}_{88}
\end{array}\right]
$$

with 


$$
\begin{gathered}
\hat{\Pi}_{12}=-U+P^{T} \hat{J}^{T} A_{1}-M_{1}+J^{T} M_{2}^{T}, \hat{\Pi}_{22}=-\left(1-\tau_{2}\right) Q_{1}+U_{2}^{T} U_{2}-\operatorname{sym}\left\{M_{2}\right\}, \\
\hat{\Pi}_{17}=-P^{T} \hat{J}^{T} H C_{h}+J^{T} C_{2}^{T} V^{T} V C_{h}, \hat{\Pi}_{77}=-I+C_{h}^{T} V^{T} V C_{h}, \hat{\Pi}_{18}=P^{T} \hat{J}^{T} \hat{B}+J^{T} C_{2}^{T} V^{T} V \hat{D}, \\
\hat{\Pi}_{33}=-\left(1-d_{2}\right) Q_{2}+U_{3}^{T} U_{3}, \hat{\Pi}_{78}=C_{h}^{T} V^{T} V \hat{D}, \hat{\Pi}_{88}=-I+\hat{D}^{T} V^{T} V \hat{D}, \\
\hat{\Pi}_{11}=\operatorname{sym}\left\{P^{T} \bar{A}\right\}+\operatorname{sym}\left\{\left(U-P^{T} \hat{J}^{T} A_{1}\right) J-M_{1} J\right\}+\tau_{1} H_{1}+\operatorname{diag}\left\{Q_{1}+C_{1}^{T} C_{1}+C_{2}^{T} V^{T} V C_{2}+U_{1}^{T} U_{1}+U_{4}^{T} U_{4}, \Lambda_{2}\right\} .
\end{gathered}
$$

Thus, if the inequality

$$
\Pi+\tau_{1} M Q_{4}^{-1} M^{T}<0
$$

holds, it follows from $\left.J[e(t), \hat{w}(t)]\right|_{\hat{w}(t) \equiv 0} \leq 0$ that $\frac{d}{d t} V(t) \leq 0$ or $V(t) \leq V(0)$. Then, from (11), it can be deduced

$$
\begin{aligned}
V(0) & =e(0)^{T} P_{1} e(0)+\int_{-\tau(0)}^{0} e(s)^{T} Q_{1} e(s) d s+\int_{-d(0)}^{0} \eta(s)^{T} Q_{2} \eta(s) d s+\int_{-\tau_{1}}^{0} \int_{s}^{0} \eta(\theta)^{T}\left(Q_{3}+Q_{4}\right) \eta(\theta) d \theta d s \\
& \leq \lambda_{\max }\left(P_{1}\right)\|\varphi\|_{2}^{2}+\lambda_{\max }\left(Q_{1}\right) \int_{-\tau(0)}^{0} e(s)^{T} e(s) d s+\lambda_{\max }\left(Q_{2}\right) \int_{-d(0)}^{0} \eta(s)^{T} \eta(s) d s+\lambda_{\max }\left(Q_{3}+Q_{4}\right) \int_{-\tau_{1}}^{0} \int_{s}^{0} \eta(\theta)^{T} \eta(\theta) d \theta d s \\
& \leq \sigma_{1}\|\varphi\|_{2}^{2}+\sigma_{2}\|\eta\|_{2}^{2}
\end{aligned}
$$

where $\sigma_{1}:=\lambda_{\max }\left(P_{1}\right)+h_{1} \lambda_{\max }\left(Q_{1}\right)$ and $\sigma_{2}:=\left(d_{1} \lambda_{\max }\left(Q_{2}\right)+0.5 h_{1}^{2} \lambda_{\max }\left(Q_{3}+Q_{4}\right)\right)$. Then, we have:

$$
\lambda_{\min }\left(P_{1}\right)\|\varphi\|_{2}^{2} \leq V(t) \leq \sigma_{1}\|\varphi\|_{2}^{2}+\sigma_{2}\|\eta\|_{2}^{2} .
$$

Therefore, we conclude that the error dynamics (6a) are asymptotically stable. Notice that the matrix inequality (25) includes multiplication of filter matrices and Lyapunov matrices which are unknown and occur in nonlinear fashion. Hence, the inequality (25) cannot be considered an LMI problem. In the literature, more attention has been paid to the problems having this nature, which called bilinear matrix inequality (BMI) problems [38]. In the sequel, by considering $P_{3}=\varepsilon P_{2}$, introducing change of variable $\hat{H}=P_{2}^{T} H$ and applying Schur Complement Lemma, the matrix inequality (25) is converted into LMI (13a) and can be solved via convex optimization algorithms. It is also easy to see that the inequality (25) implies $\hat{\Pi}_{11}<0$. Hence by Proposition 4.2 in [14], the matrix $P$ is nonsingular. Then, according to the structure of the matrix $P$ in (12), the matrix $P_{2}$ is also nonsingular.

Remark 3. It is worth noting that the LMIs (13) are linear in the set of matrices $P_{2}, V, \hat{H}, U, M_{1}, \cdots, M_{8}$, $P_{1}, H_{1}, Q_{1}, \cdots, Q_{4}$ and the scalar $\gamma^{2}$. This implies that the scalar $\gamma^{2}$ can be included as one of the optimization variables in LMIs (13) to obtain the minimum disturbance attenuation level. Then, the optimal solution to the RFDF design can be found by solving the following convex optimization problem

$$
\operatorname{Min} \lambda
$$

subject to LMIs (13) with $\lambda:=\gamma^{2}$. 
Remark 4. If we are interested in further simplification in the structure of the system (1), the nonlinear perturbations $h_{i}(t, x()$.$) can be eliminated from the system in (1) by considering h_{i}(t, x().) \equiv 0$, or equivalently $\psi_{i}(t, e().) \equiv 0$, then the LMI (13a) can be simplified to yield the following LMI

$$
\left[\begin{array}{ccccc}
\Pi_{11} & \Pi_{12} & \Pi_{13} & \Pi_{18} & J^{T} C_{2}^{T} V^{T} \\
* & \Pi_{22} & -M_{3}^{T} & -M_{8}^{T} & 0 \\
* & * & \Pi_{33} & 0 & 0 \\
* & * & * & -I & \hat{D}^{T} V^{T} \\
* & * & * & * & -I
\end{array}\right]<0
$$

In this case, the matrices $M_{4}, M_{5}, M_{6}, M_{7}$ are eliminated from the LMI variables in Theorem 1.

Remark 5. (Polytopic uncertainty) Consider the matrices of the system (1a) are not exactly known, except that they are within a compact set $\Omega=\left[C, A_{1}, A_{2}, B, E_{h_{1}}, E_{h_{2}}, E_{h_{3}}, E_{f}, B_{w}\right]$, we assume that $\Omega=\sum_{j=1}^{N} s_{j} \Omega_{j}$ for some scalars $s_{j}$ satisfying $0 \leq s_{j} \leq 1, \quad \sum_{j=1}^{N} s_{j}=1$, where the $N$ vertices of the polytope are described by $\Omega_{j}=\left[C^{(j)}, A_{1}^{(j)}, A_{2}^{(j)}, B^{(j)}, E_{h_{1}}^{(j)}, E_{h_{2}}^{(j)}, E_{h_{3}}^{(j)}, E_{f}^{(j)}, B_{w}^{(j)}\right]$ (see for instance [20]). In order to take into account the polytopic uncertainty in the system (1), we derive the following result from applying the same transformation that was used in deriving Theorem 1.

Theorem 2. Under A1)-A2), for given scalars $\gamma, \tau_{1}, d_{1}>0, \tau_{2}, d_{2}, \varepsilon$, if the uncertainty set $\Omega$ is polytopic with vertices $\Omega_{j}, j=1,2, \cdots, N$, then the system described by (1)-(2) and the RFDF (4) is asymptotically stable and satisfies the $H_{\infty}$ performance condition, simultaneously, in the sense of Definition 2 if there exist some matrices $P_{2}, V, \hat{H}, U, M_{1}, \cdots, M_{8}$ and positive-definite matrices $P_{1}, H_{1}, Q_{1}, \cdots, Q_{4}$ such that LMIs (13) are satisfied for all

$$
\left[C, A_{1}, A_{2}, B, E_{h_{1}}, E_{h_{2}}, E_{h_{3}}, E_{f}, B_{w}\right]=\left[C^{(j)}, A_{1}^{(j)}, A_{2}^{(j)}, B^{(j)}, E_{h_{1}}^{(j)}, E_{h_{2}}^{(j)}, E_{h_{3}}^{(j)}, E_{f}^{(j)}, B_{w}^{(j)}\right], \quad j=1,2, \cdots, N
$$

Then, the matrix $H$ of the RFDF can be found by computing $H:=\left(P_{2}^{T}\right)^{-1} \hat{H}$.

Proof. It follows directly from the proof of Theorem 1 and using properties of polytopic uncertainties in Remark 5.

Remark 6. The reduced conservatism of Theorems 1-2 benefit from the construction of the LyapunovKrasovskii functional in (11), introducing some free weighting matrices to express the relationship among the system matrices, utilizing the Leibniz-Newton formula for nonlinear perturbation functions in (7) and neither the model transformation approach nor any bounding technique are needed to estimate the inner 
product of the involved crossing terms (see for instance [6], [14] and [20]). It can be easily seen that results of this paper is quite different from existing results in [1] and [5] in the following perspectives: a) the structures in [1] and [5] consider, respectively, a retarded time-delay systems (with a constant discrete delay) and a delay-free linear parameter-varying system and in compare to our case do not center on mixed time-delays, i.e., the results in [1] and [5] can not be directly applied to the systems with different neutral and discrete delays and nonlinear perturbations. b) in this paper, the derived sufficient conditions are convex and neutral-delay-dependent and discrete-delay-dependent, which make the treatment in the present paper more general with less conservative in compare to the results in [1] which are independent of the discrete delay.

\section{EXAMPLE}

In this section, we will verify the proposed methodology by giving an illustrative example. We solved LMIs (13) by using Matlab LMI Control Toolbox [15], which implements state-of-the-art interior-point algorithms and is significantly faster than classical convex optimization algorithms. The example is given below.

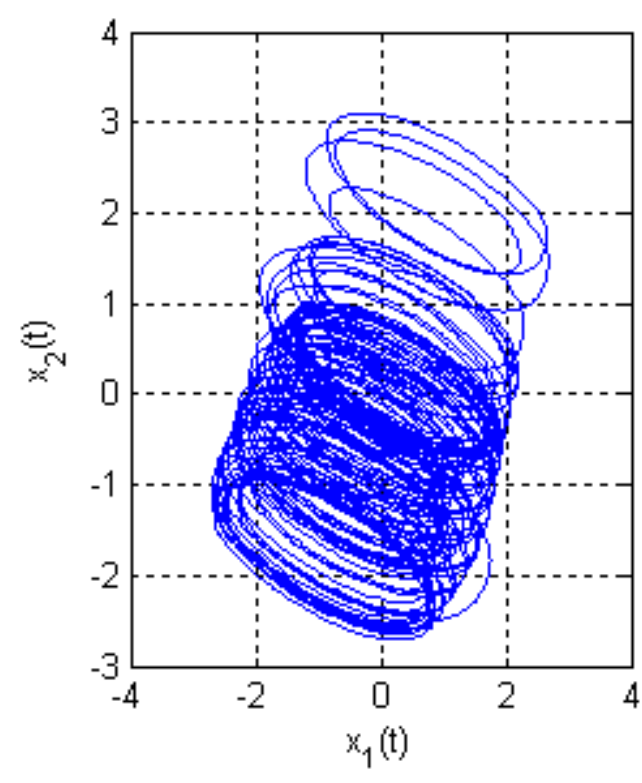

(a)

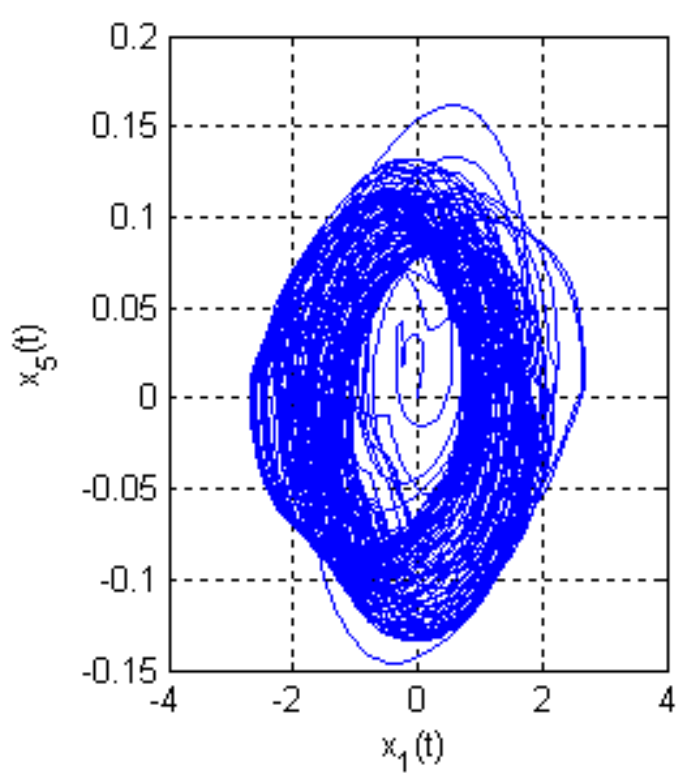

(b)

Fig. 1. The phase trajectories: a) plot $x_{1}-x_{2}$, b) plot $x_{1}-x_{5}$.

Consider the system (1) with the following state-space matrices for an aircraft model 


$$
\begin{aligned}
& A=\left[\begin{array}{cccccc}
-1.0500 & -2.5500 & 0 & 0 & -169.6600 & -0.0091 \\
2.5500 & -1.0500 & 0 & 0 & 57.0900 & 0.0017 \\
0 & 0 & -77.5300 & 39.5700 & 0 & 0 \\
0 & 0 & 0 & -20.2000 & 0 & 0 \\
0 & 0 & -8.8000 & 0 & -20.2000 & 0 \\
0 & 0 & 0 & 0 & 0 & -0.1000
\end{array}\right] ; \\
& B=[0,0,0,-4.4944,0,0]^{T} ; E_{h_{1}}=E_{h_{3}}=[0,1,0,0,1,0]^{T} ; \\
& E_{h_{2}}=[1,0,0,0,1,0]^{T} ; B_{w}=[1,0,1,0,1,1]^{T} ; A_{2}=0.01 I ; \\
& E_{f}=[3.5500,-0.5500,0,0,-0.0200,0]^{T} ; A_{1}=0.01 A ; \\
& C_{2}=\left[\begin{array}{cccccc}
-0.0100 & 0.0900 & 0.0700 & 0 & 0 & 0 \\
-0.4800 & -0.5900 & 0 & 0 & -49.5100 & -0.0026 \\
0.0300 & 0.0900 & -0.0600 & 0 & 0 & 0 \\
0.2600 & -0.0700 & 0.0100 & 0 & 0 & 0
\end{array}\right] \text {; } \\
& D=C_{h}=C_{f}=D_{w}=0 ; C_{1}=10^{3} I ; h_{i}(t, x(t))=0.5(|x(t)+1|-|x(t)-1|), \quad(i=1,2, \ldots, 4) \text {. }
\end{aligned}
$$

The delays $\tau(t)=d(t)=\left(1-e^{-t}\right) /\left(1+e^{-t}\right)$ are time varying and satisfy $0 \leq \tau(t)=d(t) \leq 1$ and $\dot{\tau}(t)=\dot{d}(t) \leq 0.5$.

With the above parameters, the model exhibits the chaotic behaviours such the phase trajectories of the system are depicted in Figure 1.

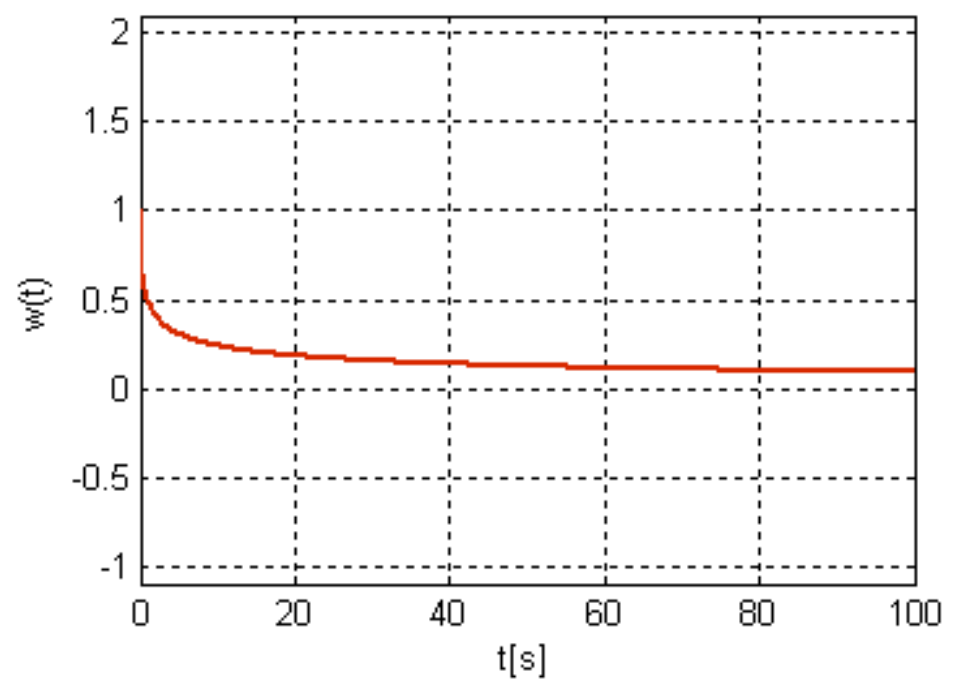

Fig. 2. Exogenous input.

With the above parameters and by Theorem 1 for $\gamma=0.25, \varepsilon=0.1$, filter gains are found by solving LMIs (13) as follows: 


$$
\begin{aligned}
& H=10^{6}\left[\begin{array}{cccc}
0.7093 & 0.0046 & -0.2267 & 1.5128 \\
-0.1254 & -0.0014 & -0.0916 & 0.0352 \\
0.3688 & -0.0059 & 0.6843 & -0.3175 \\
0.2365 & -0.02545 & -0.0065 & -0.0042 \\
-0.4174 & -0.06871 & 0.1816 & 0.2360 \\
-0.3668 & -0.0142 & 0.6843 & -0.3175
\end{array}\right] \text {, } \\
& V=10^{4}\left[\begin{array}{llll}
0.2871 & 0.0456 & -0.0015 & 0.0068
\end{array}\right] \text {. }
\end{aligned}
$$

For simulation purpose, an exogenous disturbance input, shown in Figure 2, is set as

$$
w(t)=\frac{1}{1+\sqrt{t}}, \quad t \geq 0
$$

and actuator fault modes of the elevator, i.e., step fault inputs shown in Figure 3, are imposed on the system and the control signal is assumed to be $u(t)=\sin (t)$.

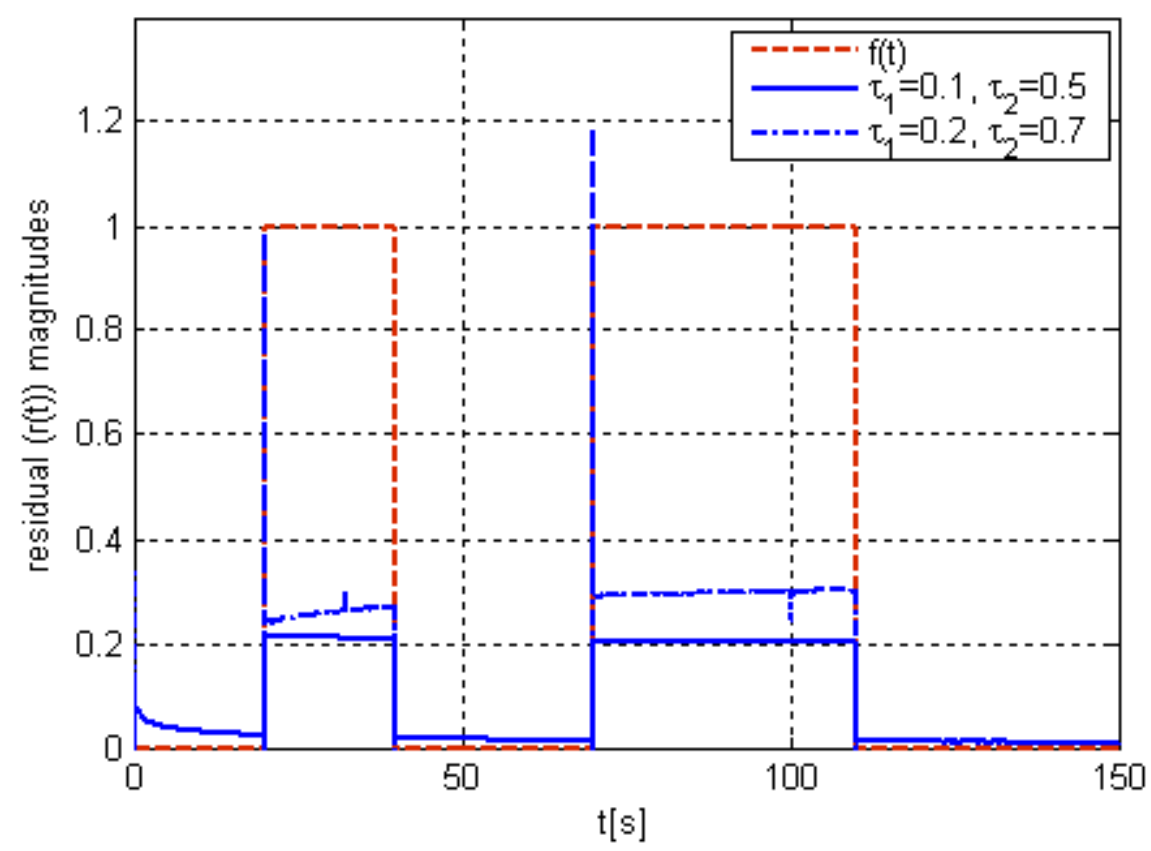

Fig. 3. Residual signals generated by RFDF.

Figure 3 shows the residual signals obtained with the filter for two different bounds of neutral delays. It can be seen that clearly by monitoring the fault estimates, it would be possible to detect fault behaviours.

Remark 7. In the case of without time-delays and nonlinear perturbations, this example, the aircraft model, was studied in the framework of a linearized parameter varying model in [5], where satisfactory results as fault detection and isolation have been obtained. In this case, the operation of the RFDF can be tested by simulation when applying, e.g., two step fault modes. In Figure 4 it can be seen that the RFDF 
follows two fault inputs more closely than the other filter in [5] and the result shows a perfect decoupling of the fault effects in the fault detection similar to the results in [5].

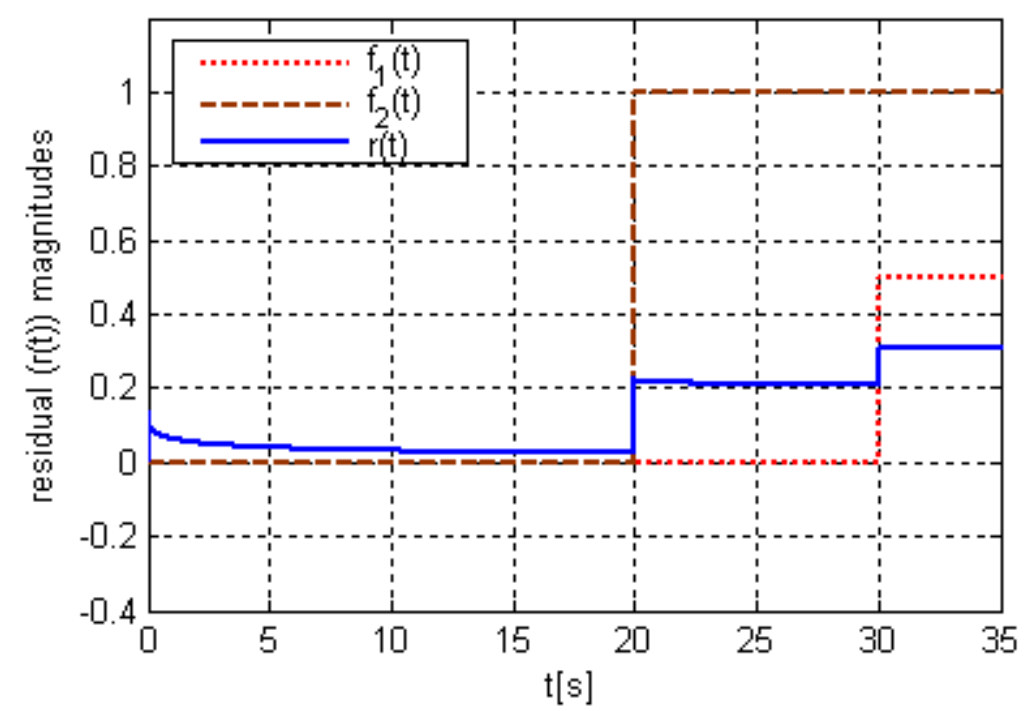

Fig. 4. Residual signals generated by RFDF in the case of $h_{i}(t, x(t))=0$ and $\tau(t)=d(t)=0$.

\section{CONCLUSION}

The problem of robust fault detection filter (RFDF) design was presented to identify faults for a class of linear systems with some nonlinear perturbations and mixed neutral and discrete time-varying delays. By using a descriptor technique, Lyapunov-Krasovskii functional and a suitable change of variables, new required sufficient conditions were established in terms of delay-dependent linear matrix inequalities to synthesize the residual generation scheme. Based on Luenberger type observers, the explicit expression of the filters was derived for the fault such that both asymptotic stability and a prescribed level of disturbance attenuation are satisfied for all admissible nonlinear perturbations. The existence of the RFDF is presented in terms of LMI formulation, which can be obtained conveniently by using Matlab LMI toolbox. The proposed method has proven its effectiveness in simulation of an aircraft model. The RFDF was able to quickly and correctly identify the faults.

\section{ACKNOWLEDGMENT}

This work has been partially funded by the European Union (European Regional Development Fund) and the Ministry of Science and Innovation (Spain) through the research project DPI2008-06699-C02-01 and by the Government of Catalonia through SGR00296. Mauricio Zapateiro is grateful to the FI Grant of the Department for Innovation, University and Enterprise of the Government of Catalonia (Spain). 


\section{REFERENCES}

[1] Bai L., Tian Z. \& Shi S., (2007). Robust fault detection for a class of nonlinear time-delay systems,' J. of the Franklin Institute, 344, 873-888.

[2] Bai L., Tian Z., \& Shi S., (2006). Design of $H_{\infty}$ robust fault detection filter for linear uncertain time-delay systems. ISA Transactions, 45(4), 491-502.

[3] Basin M., Rodriguez-Gonzalez J., \& Martinez-Zuniga R., (2004). Optimal control for linear systems with time delay in control input. J. of the Franklin Institute, 341, 267-278.

[4] Basin M. \& Rodkina A., (2008). On delay-dependent stability for a class of nonlinear stochastic systems with multiple state delays. Nonlinear Analysis, 68, 2147-2157.

[5] Bokor J. \& Balas, G. (2004). Detection filter design for LPV systems-a geometric approach. Automatica, 40, 511-518.

[6] Chen, J. D., Lien, C. H., Fan, K. K., \& Chou, J. H., (2001). Criteria for asymptotic stability of a class of neutral systems via a LMI approach. IEE Proc. Control Theory and Applications, 148, 442-447.

[7] Darouach M., (2005) 'Reduced order observer for linear neutral delay systems,' IEEE Trans Automatic Control, 50, 1407-1413.

[8] Demetriou, M. A., \& Polycarpou, M. M. (1998). Incipient fault diagnosis of dynamical systems using online approximators. IEEE Trans. Automatic Control, 43, 1612-1617.

[9] Ding, S. X., Jeinsch, T., Frank, P. M., \& Ding, E. L. (2000). A unified approach to the optimization of fault detection systems. Int. J. Adaptive Control and Signal Processing, 14, 725-745.

[10] Ding, S. X., Zhong, Maiying, Tang, Bingyong, \& Zhang, P. (2001). An LMI approach to the design of fault detection filter for time-delay LTI systems with unknown inputs. Proc. American Control Conference (21372142).

[11] Frank, P. M. (1990). Fault diagnosis in dynamic systems using analytical and knowledge-based redundancy: A survey and some new results. Automatica, 26, 459-474.

[12] Frank, P. M., \& Ding, X. (1997). Survey of robust residual generation and evaluation methods in observerbased fault detection systems. J. Process Control, 7, 403-424.

[13] Fridman, E., (2001). New Lyapunov-Krasovskii functionals for stability of linear retarded and neutral type systems. Systems \& Control Letters, 43, 309-319.

[14] Fridman, E., \& Shaked, U., (2003). Delay-dependent stability and $H_{\infty}$ control: constant and time-varying delays. Int. J. Control, 76, 48-60, 2003.

[15] Gahinet P., Nemirovsky A., Laub A.J. \& Chilali M., (1995). LMI control Toolbox: For use with Matlab. Natik, MA: The MATH Works, Inc.

[16] Gao H., Chen T., \& Lam J., (2008). A new delay system approach to network-based control, Automatica, vol. 44, no. 1, pp. 39-52. 
[17] Gao H. \& Wang C., (2003a). Delay-dependent robust $H_{\infty}$ and $L_{2}-L_{\infty}$ filtering for a class of uncertain nonlinear time-delay systems. IEEE Trans. Automatic Control, 48(9), 1661-1666.

[18] Gao, H., \& Wang, C., (2003b). Comments and further results on 'A descriptor system approach to $H_{\infty}$ control of linear time-delay systems. IEEE Trans. Automatic control, 48, 520-525.

[19] Gao H. \& Wang C., (2004). A delay-dependent approach to robust $H_{\infty}$ filtering for uncertain discrete-time state-delayed systems. IEEE Trans. Signal Processing, 52(6), 1631-1640.

[20] Gu K., Kharitonov V. L., \& Chen J., (2003). Stability of time-delay systems’ Birkhauser, Boston, ISBN 08176-4212-9.

[21] Hale, J. K., \& Verduyn Lunel, S. M. (1993). Introduction to functional differential equations. New York: Springer-Verlag.

[22] Hallouzi R., Verdult V., Babuska R., Verhaegen M., (2005). Fault detection and identification of actuator faults using linear parameter varying models. Proc. of 2005 IFAC World Congress, Prague.

[23] Han, C.L. \& Yu, L., (2004). Robust stability of linear neutral systems with nonlinear parameter perturbations. IEE Proc. Control Theory Appl., 151(5), 539-546.

[24] Jiang, B., Staroswiecki, M., \& Cocquempot, V. (2002). Fault identification for a class of time-delay systems. Proc. American Control Conference (2239-2244).

[25] Jiang C., D.H. Zhou, (2005). Fault detection and identification for uncertain linear time-delay systems. Computers and Chemical Eng., 30, 228-242.

[26] Karimi H.R., (2008). Observer-based mixed $H_{2} / H_{\infty}$ control design for linear systems with time-varying delays: An LMI approach. Int. J. Control, Automation, and Systems, 6(1), 1-14.

[27] Karimi H.R., \& Gao H., (2008). LMI-based delay-dependent mixed $H_{2} / H_{\infty}$ control of second-order neutral systems with time-varying state and input delays. ISA Transactions, 47(3), 311-324.

[28] Karimi, H. R., Zapateiro M., \& Luo N., (2008). Robust mixed $H_{2} / H_{\infty}$ delayed state-feedback control of neutral delay systems with time-varying delays. Asian J. Control, 10(5), 571-582.

[29] Krasovskii, N.N., (1963). Stability of motion. Stanford, CA: Stanford University Press.

[30] Kuang, Y., (1993). Delay differential equations with applications in population dynamics. Acadelic Press, Boston.

[31] Lien, C.H., (2005). Guaranteed cost observer-based controls for a class of uncertain neutral time-delay systems. J. Optimization Theory and Applications, 126(1), 137-156.

[32] MacDonald, N., (1989). Biological delay systems: linear stability theory. Cambridge University Press, Cambridge.

[33] Malek-Zavarei, M., \& Jamshidi, M., (1987). Time-delay systems: Analysis, optimisation and application. Amsterdam, The Netherlands: North-Holland.

[34] Meskin N., \& Khorasani K., (2009) 'Robust fault detection and isolation of time-delay systems using a geometric approach’, Automatica, 45, 1567-1573. 
[35] Niculescu S. I., (2001). Delay Effects on Stability: A Robust Control Approach. (Berlin: Springer).

[36] Park P., (1999). A delay-dependent stability criterion for systems with uncertain time-invariant delays. IEEE Trans. Autom. Control, 44, 876-877.

[37] Patton R.J., Frank P.M., \& Clark R.N., (2000). Issues of Fault Diagnosis for Dynamic Systems. Verlag: Springer, Berlin.

[38] Safonov M.G., Goh K.C., \& Ly J.H., (1994). Control system synthesis via bilinear matrix inequalities. Proc. ACC., 45-49.

[39] de Souza, C.E., Palhares, R.M., Peres, P.L.D., (2001). Robust $H_{\infty}$ filter design for uncertain linear systems with multiple time-varying state delays. IEEE Trans. Signal Processing, 49, 569-576.

[40] Trunov, A. B., \& Polycarpou, M. M. (2000). Automated fault diagnosis in nonlinear multivariable systems using a learning methodology. IEEE Transactions on Neural Networks, 11, 91-101.

[41] Vemuri, A. T., \& Polycarpou, M. M. (1997). Robust nonlinear fault diagnosis in input-output systems. Int. J. Control, 68, 343-360.

[42] Venkatasubramanian, V., Rengaswamy, R., Yin, K., \& Kavuri, S. N. (2003). A review of process fault detection and diagnosis Part I: Quantitative model-based methods. Computers and Chemical Eng., 27, 293311.

[43] Wang, H. \& Daley, S. (1996). Actuator fault diagnosis: An adaptive observer-based technique. IEEE Trans. Autom. Control, 41, 1073-1078.

[44] Wang, H., Huang, Z. J., \& Daley, S. (1997). On the use of adaptive updating rules for actuator and sensor fault diagnosis. Automatica, 33, 217-225.

[45] Xu S., \& Chen T., (2004) 'An LMI approach to the $H_{\infty}$ filter design for uncertain systems with distributed delay,' IEEE Trans. Circuits and Systems II, 51, 195-201.

[46] Xu, S., Lam, J., \& Yang, C., (2001). $H_{\infty}$ and positive-real control for linear neutral delay systems. IEEE Trans. Automa. Contr., 46, 1321-1326.

[47] Yang, Hanlong, \& Saif, Mehrdad. (1998). Observer design and fault diagnosis for state-retarded dynamical systems. Automatica, 34, 217-227.

[48] Yue, D., Han, Q.L., \& Lam, J., (2005) 'Network-based robust $H_{\infty}$ control of systems with uncertainty,' Automatica, 41(6), 999-1007.

[49] Zhang, P., Ding, S. X., Wang, G. Z., \& Zhou, D. H. (2002). Fault detection for multirate sampled-data systems with time-delays. Int. J. Control, 75, 1457-1471.

[50] Zhong M., Ding S.X., Lam J. \& Wang H., (2003). An LMI approach to design robust fault detection filter for uncertain LTI systems. Automatica, 39, 543 - 550.

[51] Zhou K. \& Khargonekar P.P., (1988). Robust stabilization of linear systems with norm-bounded time-varying uncertainty. System Control Letters, 10, 17-20. 